\title{
Cholesterol's Location in Lipid Bilayers
}

\author{
Drew Marquardt ${ }^{a, b, *}$, Norbert Kučerkac,d, Stephen R. Wassalle, Thad A. Harroun ${ }^{\mathrm{f}}$, John \\ Katsaras $^{\mathrm{f}, \mathrm{g}, \mathrm{h}, \mathrm{i}, *}$ \\ ${ }^{a}$ University of Graz, Institute of Molecular Biosciences, Biophysics Division, NAWI Graz, Humboldtstr. \\ 50/III, 8010 Graz, Austria \\ ${ }^{b}$ BioTechMed-Graz, Austria. \\ ${ }^{c}$ Frank Laboratory of Neutron Physics, Joint Institute for Nuclear Research, 141980 Dubna - Moscow \\ Region, Russia \\ ${ }^{d}$ Department of Physical Chemistry of Drugs, Faculty of Pharmacy, Comenius University, 83232 \\ Bratislava, Slovakia \\ ${ }^{e}$ Department of Physics, Indiana UniversityPurdue University Indianapolis, Indianapolis, Indiana 46202, \\ United States \\ ${ }^{f}$ Department of Physics, Brock University, St. Catharines, Ontario L2S 3A1, Canada. \\ ${ }^{g}$ Oak Ridge National Laboratory, Oak Ridge, Tennessee 37831, United States. \\ ${ }^{h}$ Joint Institute for Neutron Sciences, Oak Ridge, Tennessee 37831, United States. \\ ${ }^{i}$ Department of Physics, University of Tennessee, Knoxville, Tennessee 37996, United States.
}

\begin{abstract}
It is well known that cholesterol modifies the physical properties of lipid bilayers. For example, the much studied liquid-ordered $\mathrm{L}_{o}$ phase contains rapidly diffusing lipids with their acyl chains in the all trans configuration, similar to gel phase bilayers. Moreover, the $L_{o}$ phase is commonly associated with cholesterol-enriched lipid rafts, which are thought to serve as platforms for signaling proteins in the plasma membrane. Cholesterol's location in lipid bilayers has been studied extensively, and its been shown - at least in some bilayers to align differently from its canonical upright orientation, where its hydroxyl group is in the vicinity of the lipid-water interface. In this article we review recent works describing cholesterol's location in different model membrane systems with emphasis on results obtained from scattering, spectroscopic and molecular dynamics studies.
\end{abstract}

Keywords: cholesterol, sterol, membrane dynamics, membrane structure, lipid domains 2010 MSC: 00-01, 99-00

\footnotetext{
Fully documented templates are available in the elsarticle package on CTAN.

*Corresponding author

Email addresses: drew.marquardt@uni-graz.at (Drew Marquardt), katsarasj@ornl.gov (John Katsaras)
} 


\section{Introduction}

Few biomolecules have been scrutinized as much as cholesterol. Numerous works have been written about its life-cycle in the human body, its association with other biomolecules, and its role in human health (Myant, 1981). Moreover, many interesting physical properties have been attributed to it. Although a focused review, as this, cannot do proper justice to cholesterol's biological importance, we will focus on recent results specifying cholesterol's location in different model membranes, with special emphasis on data derived from scattering, spectroscopic and molecular dynamics studies.

Cholesterol is found in all animal cell membranes and is needed for their proper function, including membrane permeability and fluidity (Smith, 1991; Parasassi et al., 1995). It is also thought to act as an antioxidant, and has been implicated in cell signaling processes associated with functional domains in the plasma membrane (Petrie et al., 2000; Papanikolaou et al. 2005). As much as $90 \%$ of total cholesterol is found in the plasma membrane (PM) (Lange and Ramos, 1983), and accounts for up to $45 \mathrm{~mol} \%$ of the membrane's total lipid content (Yeagle, 1993) - it should be pointed out, however, that organelle membranes are almost devoid of it (Mouritsen, 2005). Since its discovery in 1769 by F.P. de la Salle (Stillwell, 2013), cholesterol has become one of the most studied biomolecules (Brown and Goldstein, 1992). However, despite the wealth of research data, many questions remain about cholesterol's role in membranes.

Cholesterol is made up of a hydrocarbon tail, a fused planar 4-ring assembly - common to steroid hormones (i.e., testosterone and estrogen) - and a hydroxyl headgroup that helps it orient at the membrane-water interface. Although a planar molecule, cholesterol has methyl groups on its two quaternary centres, making up its so-called "rough" or $\beta$ face, while their absence on the molecule's other side account for its "smooth" or $\alpha$ face (Figure 1). Although there are different cholesterol isoforms (i.e., 256), only one is naturally occurring (i.e., SRSSSRRR-cholesterol) (Westover et al., 2003, Kristiana et al., 2012).

In water cholesterol aggregates, forming micelles at concentrations between 20 and 40 nM (Haberlan and Reynolds, 1973; Gilbert et al., 1975). However, due to its hydrophobic nature it has a solubility of only $1.8 \mathrm{mg} / \mathrm{L}$ Haberlan and Reynolds (1973). Cholesterol excluded from the membrane adopts a crystalline monohydrate form (Brzustowicz et al. 

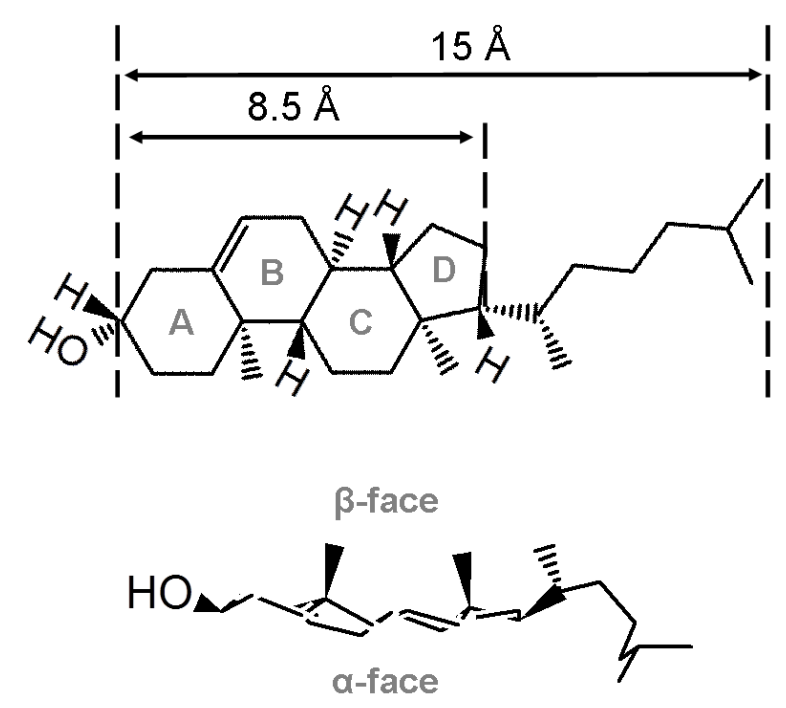

Figure 1: (a) The structure of cholesterol showing its steroid skeleton of four fused rings, and its fatty acid tail. Molecular dimensions were taken from Marquardt et al. (2015d); Kučerka et al. (2008). (b) Schematic showing differences between the molecule's $\alpha$ - and $\beta$-faces. Hydrogen atoms are not shown for better viewing.

2002b). In this review we will report on cholesterol's location and influence on different lipid bilayers as studied by neutron scattering and nuclear magnetic resonance (NMR). Understanding cholesterol's influence on model membranes may eventually help us to resolve some of the open questions associated with this important biomolecule.

\section{Cholesterol in Different Bilayer Systems}

Cholesterol alters lipid membranes in complex ways, and over the years a number of experimental techniques have been used to construct phase diagrams of cholesterol-containing membranes. In this section we will review some of the experimental techniques used to determine the location of cholesterol in model membranes.

Determining cholesterol's location in model membranes has made use of a number of different sample preparations. For example, commonly used and easy to prepare multilamellar vesicles (MLVs) have the advantage of being easily hydrated under different buffer conditions. Unilamellar vesicles (ULVs) are in many ways similar to MLVs, but with the addition that they have to be extruded or sonicated from a parent MLV solution. An advantage of ULVs is that when extruded, their size is well defined, allowing for studies probing the effects of curvature on membrane organization. Model membranes can also be aligned on 
rigid substrates (e.g., using single crystal silicon or glass), forming smectic layers. Breaking the symmetry allows the experiment to probe orientation with respect to the bilayer normal, but also allows for a better signal-to-noise ratio.

\subsection{Scattering}

Elastic neutron and x-ray scattering have been used to determine the location of cholesterol in model membrane systems (Worcester and Franks, 1976; Franks and Lieb, 1979). A thorough list of literature determining the location cholesterol via scattering can be found in Marquardt and Harroun (2014). Neutron scattering is a particularly powerful method for examining materials inherently rich in hydrogen, due to the neutron's ability to distinguish between hydrogen $\left({ }^{1} \mathrm{H}\right)$ and deuterium $\left({ }^{2} \mathrm{H}\right)$ atoms - e.g., $63 \mathrm{~mol} \%$ of cholesterol is hydrogen. Individual or groups of hydrogen atoms within a molecule can selectively be replaced with deuterium, and because of the large difference in neutron scattering power between ${ }^{2} \mathrm{H}$ and ${ }^{1} \mathrm{H}$, this substitution provides the necessary contrast needed to locate groups of interest. For example, the difference in measured scattering between ${ }^{2} \mathrm{H}$-cholesterol and ${ }^{1} \mathrm{H}$-cholesterol identifies the location and distribution of the ${ }^{2} \mathrm{H}$ label within the membrane (Harroun et al. 2006a, 2008; Kučerka et al., 2010).

$\mathrm{X}$-ray scattering is in many ways a complementary technique to neutron scattering. Compared to neutrons, X-ray sources offer more intense beams, greater instrumental resolution, and wavelength spreads that are approximately 2 orders of magnitude finer (Marquardt et al. 2015b). These features allow for better data quality through significantly sharper peaks and a better signal-to-noise, especially at higher scattering angles, which translates in to higher resolution scattering density profiles of the membrane. For a detailed review of scattering on membranes see Marquardt et al. (2015b).

\subsection{Nuclear Magnetic Resonance}

Nuclear magnetic resonance (NMR) spectroscopy is a technique that can be used in a number of different ways, allowing for studies of cholesterol in model membranes under biologically relevant conditions. Like neutron scattering, isotopic enrichment is used to "highlight" a specific functional moiety in a biomolecule. For example, analysis of solid state ${ }^{2} \mathrm{H}$ NMR spectra of deuterated lipid analogs have yielded the fluctuation and orientation of 
acyl chain C- ${ }^{2} \mathrm{H}$ bonds with respect to the bilayer normal (Seelig, 1977; Davis, 1983). How cholesterol orients in different membranes has also been mapped with phospholipid analogs having selectively deuterated and perdeuterated acyl chains (Vist and Davis, 1990: Yasuda et al., 2014; Mihailescu et al., 2011), while cholestrol tilt in a membrane has been found to be responsive to lipid environments with deuterated analogs of cholesterol Brzustowicz et al. 1999; Shaikh et al., 2006)

The order parameter is the quantity most often measured in ${ }^{2} \mathrm{H}$ NMR studies. It is defined according to

$$
S_{C D}=\frac{1}{2}\left\langle 3 \cos ^{2} \beta-1\right\rangle
$$

where $\beta$ is the angle for a $\mathrm{C}^{2} \mathrm{H}$ bond relative to the bilayer normal, which constitutes the axis of motional averaging for the reorientation of lipid molecules, and the angular brackets designate a time average (Seelig, 1977). The value of the order parameter describes the degree of anisotropy of molecular motion at the site of isotopic substitution. Values typically fall in the range $0 \leq \mathrm{S}_{\mathrm{CD}} \leq \frac{1}{2}$ for methylene segments labeled on a phospholipid chain, the lower limit corresponding to isotropic motion and the upper limit corresponding to fast axial rotation in the all-trans configuration. However, this interpretation must be modified in cases when the most probable orientation for a $\mathrm{C}^{2} \mathrm{H}$ bond is not perpendicular to the bilayer normal, due to conformational constraints such as on labeled sites in the vicinity of a double bond in an unsaturated phospholipid chain (Seelig and Waespe-Sarcevic, 1978) or in the rigid steroid moiety of cholesterol Marsan et al. (1999).

\subsection{Molecular Dynamics Simulations}

The molecular origin of how cholesterol affects lipids has been extensively probed by molecular dynamics (MD) simulations, both from a structural and thermodynamic perspective (Chiu et al., 2002; Hofsäß et al., 2003; Róg and Pasenkiewicz-Gierula, 2003; Aittoniemi et al., 2006; Tieleman and Marrink, 2006; Zhang et al., 2008; Kučerka et al., 2008; Bennett et al., 2009; Khelashvili and Harries, 2013). With MD simulation, the location, orientation and putative energy of cholesterol in different environments can be monitored over timescales as long as microseconds. Unique to MD simulations, molecular interactions are analyzed on an individual molecular basis, rather than by measuring macroscopic sample averages, and 
individual events can be directly observed, rather than making inferences of actions that are too fast to measure.

\section{Cholesterol's Location in Phospholipid Bilayers}

As mentioned, a number of experimental techniques have been used to determine cholesterol's location in lipid bilayers (Marquardt and Harroun, 2014). Early and subsequent studies have determined cholesterol's hydroxyl group locating near the lipid-water interface with its acyl chain buried deep in the bilayer's hydrocarbon region - this is the canonical upright orientation (Franks and Lieb, 1979; Dufourc et al., 1984; Leonard et al., 2001; Kessel et al., 2001; Harroun et al., 2006a, 2008; Shrivastava et al., 2009; Subczynski et al., 2009; Kučerka et al., 2010). This arrangement has been associated with cholesterol's ability to modulate membrane properties by steric interactions with the lipid's acyl chains. In particular, cholesterol affects the lipids transmembrane thickness, lateral area and headgroup hydration monotonically as a function of the biomolecule's concentration - up to $45 \mathrm{~mol}$ \% (Kučerka et al., 2007; Pan et al., 2008). Acyl chain hydrophobic mismatch is one obvious cause of this effect. However, another reason for bilayer thickening is the well known condensation effect induced by cholesterol (Róg and Pasenkiewicz-Gierula, 2001), whereby it increases the lipid hydrocarbon chain order. In fact, this interaction appears to be more profound when associated with hydrophobic matching, as bilayer thickening has also been reported for long-chain lipids (Kučerka et al., 2008). Interestingly, this implies that cholesterol's ordering effect on lipid hydrocarbon chain organization dominates over the ability of long-chain lipid membranes to reduce their hydrophobic mismatch. In other words, it is more favourable for cholesterol to order acyl chains and thicken membranes, rather than reduce the hydrophobic mismatch.

Compared to ester lipids, Pan et al. have determined a different mode of interaction between cholesterol and ether lipids - while maintaining its upright orientation. In ether bilayers, cholesterol is found to reside higher in the membrane and hydrogen bonds with the phosphate oxygens, behaviors that can be attributed to the absence of the ester oxygens (Pan et al., 2012). Although this change in location is only a few angstroms difference, the end result is that the ether lipid headgroups are less hydrated and the hydrocarbon chain packing 
is altered (again, compared to ester lipids), such that it causes cholesterol to tilt (Pan et al. 2012).

\subsection{Cholesterol Ordering of Membranes}

The ordering effect that cholesterol has on lipid bilayers is best observed by ${ }^{2} \mathrm{H}-\mathrm{NMR}$ experiments, that determine acyl chain order parameters in essentially, a non-invasive manner. A signature profile of order parameter exists along the saturated chains of phospholipids in the lamellar liquid crystalline phase (Seelig, 1977). As exemplified by the smoothed profile generated for the perdeuterated palmitoyl sn-1 chain in 1- $\left[{ }^{2} \mathrm{H} 31\right]$ palmitoyl2-oleoylphosphatidylcholine (POPC-d31), there is a plateau region of slowly varying order $\left(\mathrm{S}_{C D} \sim 0.2\right)$ in the top half of the chain followed by a progressively more rapid drop-off in the bottom half, towards the disordered terminal methyl group $\left(\mathrm{S}_{C D} \sim 0.02\right)$ (Shaikh et al., 2015), see Figure 2.

POPC-d31 - a lipid representative of a naturally occuring membrane phospholipid exhibits a continuous increase in order that almost doubles in magnitude as a function of cholesterol content over 0-45 mol\% (Lafleur et al., 1990). This is because cholesterol's fused ring structure imposes a restriction on reorientation in the upper motion part of the phospholipid chains, that then propagates into the lower part. There are, however, differences in the magnitude of the response and its dependence upon the concentration of the sterol with different lipids.

The ordering effect exerted on the perdeuterated N-palmitoyl chain in $\left[{ }^{2} \mathrm{H}_{31}\right]-\mathrm{N}$-palmitoylsphingomyelin (PSM- $\left.\mathrm{d}_{31}\right)$ flattens off in the presence of $\geq 25 \mathrm{~mol} \%$ cholesterol (Bartels et al., 2008; Bunge et al. 2008) (Figure 2). This behavior was attributed to a more favorable interaction with the saturated sphingolipid than the monounsaturated phospholipid. Greater order is also attained in raft-like mixtures of PSM- $\mathrm{d}_{31}$ with cholesterol, where order parameters $\mathrm{S}_{C D} \geq 0.4$ within the plateau region approach the value $\left(\mathrm{S}_{C D}=0.5\right)$ for an all-trans acyl chain undergoing fast axial rotation in an idealized $\mathrm{L}_{o}$ phase. The differential in order between saturated N-stearoylsphingomyelin (SSM) and 1-palmitoyl-2-stearoylphosphatidylcholine (PSPC) bilayers containing $50 \mathrm{~mol} \%$ cholesterol, however, is small as judged by quadrupolar splittings measured for analogs of these structurally similar lipids selectively deuterated in $\mathrm{N}$-acyl and 


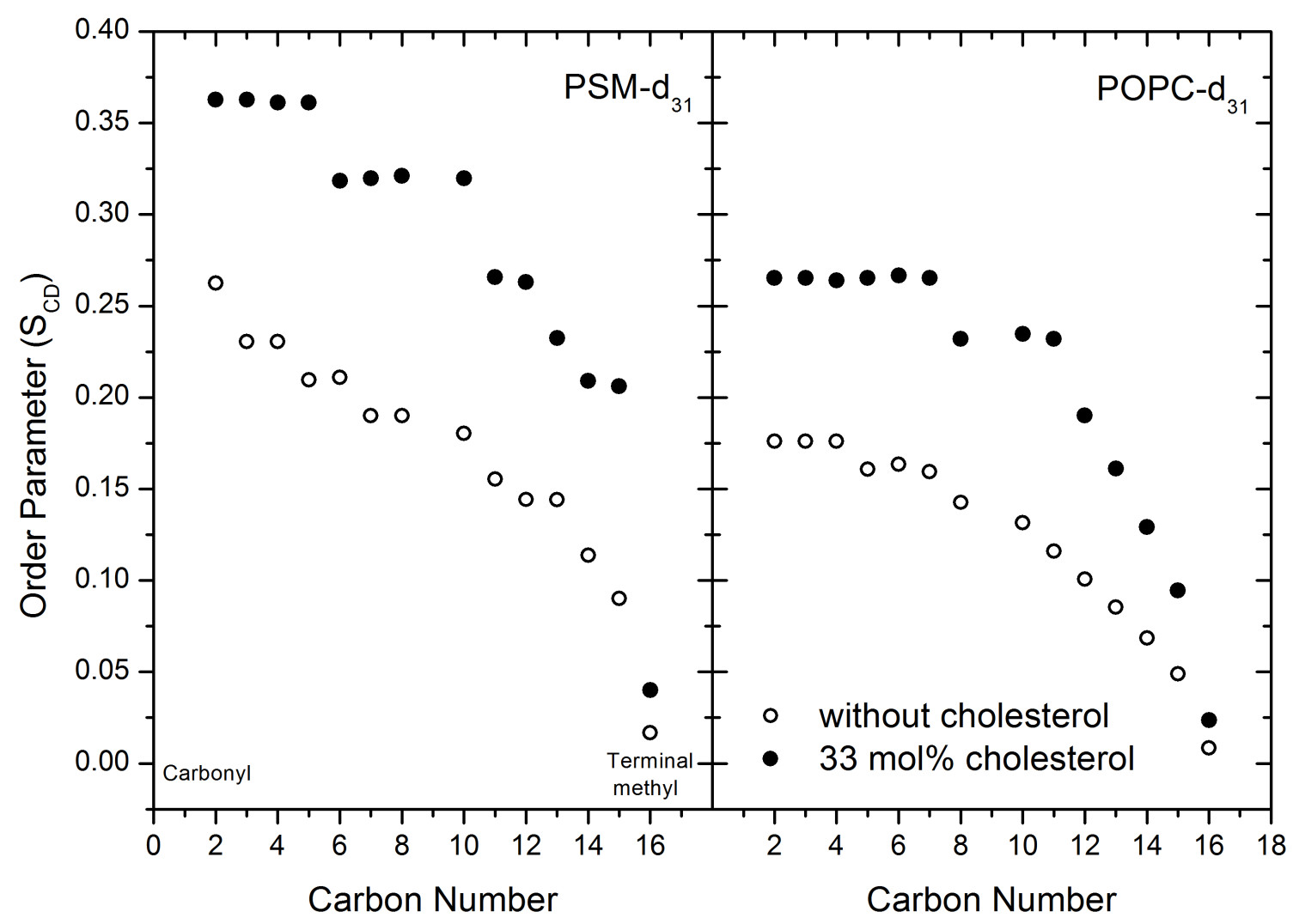

Figure 2: Order parameters $\left(\mathrm{S}_{C D}\right)$ vs carbon number for chain-deuterated PSM- $\mathrm{d}_{31}$ and POPC- $\mathrm{d}_{31}$ without (open circles) and with $33 \mathrm{~mol} \%$ cholesterol at $60{ }^{\circ} \mathrm{C}$. Note the dramatic increase in order along the fatty acid chain away from the terminal methyl. $\mathrm{S}_{C D}$ data were reproduced from Bartels et al. $(2008)$. 
sn-2 acyl chains, respectively (Yasuda et al., 2014). A less steep dependence upon temperature for quadrupolar splittings in SSM, compared to PSPC, was taken to signify greater thermal stability for SM-cholesterol bilayers, from which it was inferred that SM would be favored in rafts over saturated PC.

At the opposite extreme are polyunsaturated fatty acid (PUFA) containing phospholipids that have a low affinity for cholesterol due to the highly disordered PUFA chains Wassall and Stillwell, 2008). Less condensation of 1-[2 $\left.\mathrm{H}_{35}\right]$ stearoyl-2-docosahexaenoylphosphatidylcholine $\left(\mathrm{SDPC}-\mathrm{d}_{35}\right)$ than saturated and monounsaturated bilayers is indicated by smoothed order parameter profiles constructed for the perdeuterated stearoyl $s n-1$ chain following the addition of $29 \mathrm{~mol} \%$ cholesterol (Mihailescu et al., 2011). Reflecting a redistribution of DHA chains towards the middle of the bilayer, as identified by neutron scattering experiments, the effect is slightly more marked in the lower portion of the chain. Support for the notion that cholesterol preferentially interacts with saturated chains was derived from moment analysis of ${ }^{2} \mathrm{H}$ NMR spectra in a study of another polyunsaturated phospholipid that revealed a similar incremental increase in order for the perdeuterated palmitic acid chain in 1- $\left[{ }^{2} \mathrm{H}_{31}\right]$ palmitoyl-2-arachidonylphosphatidylcholine (PAPC- $\mathrm{d}_{31}$ ) and $1-\left[{ }^{2} \mathrm{H}_{31}\right]$ palmitoyl-2linoleoylphosphatidylcholine (PLPC- $\left.\mathrm{d}_{31}\right)$ with four and two double bonds, respectively (Jackman et al., 1999).

\subsection{Cholesterol Volumes in Lipid Bilayers}

Molecular volumes of cholesterol are required as an input for evaluating the structural details of lipid bilayers - for the detailed evaluation of x-ray or neutron scattering data, for example. The volumes of cholesterol in different lipid bilayers are listed in Table 1. The nomenclature n1:u1-n2:u2 PC is used, where $\mathrm{n} 1$ and $\mathrm{n} 2$ are the number of carbons in the $s n-1$ and $s n-2$ chains, respectively, and $\mathrm{u} 1$ and $\mathrm{u} 2$ are the number of unsaturated bonds in the respective chain. Cholesterol volumes increase significantly at concentrations beyond the liquid-disordered $\left(\mathrm{L}_{d}\right)$ phase boundary - which varies from system-to-system. The generally accepted concentration where this volume increase takes place is between 20 and $30 \mathrm{~mol} \%$ cholesterol.

From volume measurements (Greenwood et al., 2006; Gallová et al., 2015) it has been 
Table 1: Cholesterol volumes in lipid bilayers

\begin{tabular}{|c|c|c|}
\hline "Solvent" & \multicolumn{2}{|c|}{$\mathbf{V}_{\text {cho }}$} \\
\hline $\mathrm{H}_{2} \mathrm{O}$ & \multicolumn{2}{|c|}{$627^{* a}$} \\
\hline $\mathrm{H}_{2} \mathrm{O}$ & \multicolumn{2}{|c|}{$606^{* * a}$} \\
\hline di16:0ePC $\left(40^{\circ} \mathrm{C}\right)$ & \multicolumn{2}{|c|}{$676.3^{b}$} \\
\hline $\operatorname{di16}: 0 \mathrm{ePC}\left(50^{\circ} \mathrm{C}\right)$ & \multicolumn{2}{|c|}{$575.5^{b}$} \\
\hline & $\underline{\mathbf{L}_{d}}$ & $\underline{\mathbf{L}_{o}}$ \\
\hline di16:0PC & $574^{c}, 606^{a}$ & $637.1^{c}, 647.1^{a}$ \\
\hline di14:0PC & $565.1^{a}$ & $637.5^{a}$ \\
\hline sphingomylein & $575.2^{a}$ & $639.5^{a}$ \\
\hline 16:0-18:1PC & $622.6^{a}$ & \\
\hline di18:1PC & $632.9^{a, d}$ & \\
\hline $\operatorname{di} 20: 1 \mathrm{PC}$ & $627^{d}$ & \\
\hline di22:1PC & $621^{d}$ & \\
\hline di24:1PC & $620^{d}$ & \\
\hline
\end{tabular}

$a^{a}$ ref. Greenwood et al. (2006); ${ }^{b}$ ref. Pan et al. (2012); ${ }^{c}$ ref. Miyoshi et al. (2014); ${ }^{d}$ ref. Gallová et al. (2015)

surmised that cholesterol mixes ideally with monounsaturated phosphocholines in the fluid phase. Average thermal expansivity for diN:1PC with $\mathrm{N}=18,20$ and 22 is $(71.5 \pm 1.1)$ $10^{-5} \mathrm{~K}^{-1}$. The partial volume of cholesterol also decreases slightly with the lengthening of the diN:1PC host molecule (Gallová et al., 2015). Lipids with saturated chains, on the other hand, exhibit two mixing regimes, and cholesterol partial volumes clearly show the molecule's well known condensation effect (Greenwood et al., 2006).

\subsection{Cholesterol-PUFA Interaction}

Neutron scattering studies have enabled us to locate cholesterol in PUFA bilayers, namely DAPC (20:4-20:4PC). They revealed cholesterol preferentially sequestering near the bilayer center (Figure $3 \mathrm{C}$ ), in contrast to its usual location, where its hydroxyl group locates near the lipid-water interface (i.e., upright orientation, Figure 3 A). Of note were experiments detailing cholesterol's orientation in mixed lipid bilayer systems (Kučerka et al., 2009). For 


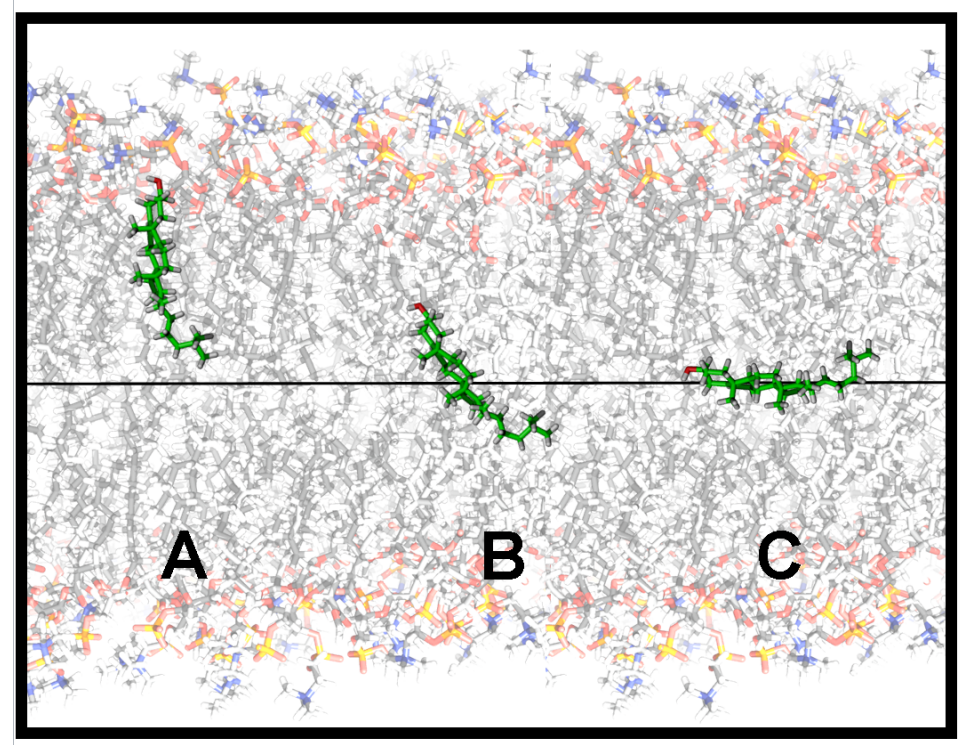

Figure 3: Using different experimental techniques (i.e., neutron scattering and NMR) and MD simulations, the location of cholesterol has been determined in different membrane environments. A) The "canonical location", B) cholesterol interdigitated between leaflets, and C) cholesterol sequestered in the center of polyunsaturated fatty acid (PUFA) bialyers.

example, when "titrating" POPC (16:0-18:1PC) into DAPC bilayers, it was found that it took $\sim 50 \mathrm{~mol} \%$ of POPC to cause cholesterol to revert to its upright orientation, while only 5 mol\% of the disaturated DMPC (14:0-14:0 PC) lipid was needed to achieve the same effect. This result is also consistent with previous data showing cholesterol's reduced solubility and binding affinity in PUFA membranes (Niu and Litman, 2002; Shaikh et al., 2006; Williams et al. 2013). The demonstration of cholesterol's affinity for lipids with saturated hydrocarbon chains, and its aversion for PUFAs, suggests the structural, and perhaps even functional, significance of lipid diversity exhibited by the lipidome.

Insensitivity to the degree of unsaturation in the $s n-2$ chain is revealed by tilt angles $\left(\alpha_{0}\right)$ calculated from order parameters measured with $\left[3 \alpha^{-2} \mathrm{H}_{1}\right]$ cholesterol - an analog labeled at the $3 \alpha$ position - incorporated at an equimolar concentration into bilayers comprised of phospholipids possessing a saturated $s n-1$ chain (Murari et al., 1986; Brzustowicz et al. 1999; Shaikh et al., 2006). The tilt angle $\alpha_{0}$, representing the most probable orientation of the long molecular axis, was extracted by invoking a Gaussian distribution to describe the angular fluctuations (Oldfield et al. 1978). A value of $\alpha_{0}=16-17^{\circ}$ was found in membranes 
ranging from 1,2-dipalmitoylphosphatidylcholine (DPPC) with saturated palmitic acid at the $s n-1$ and $s n-2$ positions to SDPC with saturated stearic acid, and polyunsaturated DHA at the $s n-1$ and $s n-2$ positions, respectively. Preferential interaction with saturated chains is implied.

The planar facade of the tetracyclic ring is, however, more compatible with close proximity to a saturated chain that adopts a largely linear configuration as opposed to, most acutely, a PUFA chain that rapidly interconverts through all conformational space (Gawrisch and Soubias, 2008). An appreciably larger tilt angle, $\alpha_{0}=24-25^{\circ}$, was determined in 1,2diarachidonylphosphatidylcholine (DAPC) and 1,2-didocosahexaenoylphosphatidylchiline (DDPC) membranes prepared from 1:1 mol mixtures of phospholipid and $\left[3 \alpha^{-}{ }^{2} \mathrm{H}_{1}\right]$ cholesterol (Brzustowicz et al., 2002a b). The aversion cholesterol has for PUFA in these membranes, where intimate contact cannot be avoided, reduces its solubility by more than a factor of 3 relative to most phospholipids, including their counterparts 1-stearoyl-2-arachidonylphosphatidylchoine (SAPC) and SDPC with a saturated $s n-1$ chain. Moreover, a non-standard location is identified by neuron scattering experiments that show both the head group and tail of cholesterol reside near the middle of DAPC bilayers (Harroun et al., 2006b, 2008). The non-standard location of cholesterol has only been observed in phospholipids where the $s n-1$ and $s n-2$ positions are populated with PUFA chains, and not in mixed chain lipids (Harroun et al., 2006b; Mihailescu et al., 2011). It should also be noted here that PUFA chains are extremely prone to the oxidation. The original studies therefore utilized various precautions to reduce lipid oxidation, including conducting the experiments in an oxygen free environment, monitoring bilayer break-down throughout the experiments, and post data collection analysis of the samples conducted to evaluate sample integrity.

Despite its aversion to PUFAs, cholesterol has been proposed as an antioxidant. From a chemical standpoint, cholesterol can serve as a free radical scavenger. Unlike $\alpha$-tocopherol whose primary function is widely thought to be the protection of PUFA containing phospholipids (Traber and Atkinson, 2007), cholesterol's hydroxyl group is not the sacrificial moiety. Instead the unsaturated bond at the $\Delta 5-6$ position on the steroid moiety is responsible for antioxidant activity (Smith, 1991; Girotti, 2001). A deeper location of cholesterol within the bilayer means that the reactive center on the sterol is better placed to intercept lipid peroxyl 
radicals than the hydroxyl group in alpha-tocopherol, which typically sits near the aqueous interface (DMPC bilayers being the exception) (Marquardt et al., 2013, 2014, 2015c). It should be borne in mind, however, that recent MD simulations reveal alpha-tocopherol can easily tunnel into the bilayer's interior, as a result of the biomolecule's methyl groups shielding its hydroxyl group (Leng et al., 2015).

Chemical activity does not necessarily signify in vivo function, as illustrated, for example, by DNA (Azzi, 2007). Smith (1991) makes several arguments for cholesterol having a protective in vivo role, namely: (i) it exists in environments which are susceptible to oxidative stress; (ii) it can be readily oxidized by reactive oxygen species; (iii) oxidized cholesterol is commonly found in vivo; and (iv) oxidized cholesterol is metabolized and excreted from the body. Other studies attribute the apparent protective properties of cholesterol purely to structural interactions and modifications induced to the bilayer (Parasassi et al. 1995 , Pandey and Mishra, 1999). Knowing the impact that cholesterol has on membrane organization, the structural interaction hypothesis seems plausible. Although much of the sterols's role in biological membranes is still not clear, it is generally accepted that when created in the distant past, cholesterol's biological function was to protect against oxygen - lung surfactant, for example, is rich in cholesterol (Brown and Galea, 2010).

\subsection{Bilayer Leaflet Distribution}

Although cholesterol's location(s) in biological membranes is now reasonably well established, its distribution between bilayer leaflets is less so. Compositional asymmetry is central for biological membranes, and it is generally accepted that cholesterol is (not surprisingly) asymmetrically distributed between bilayer leaflets (Marquardt et al., 2015a; Nickels et al., 2015). For example, plasma membrane data suggests a greater concentration of cholesterol in the cytosolic facing monolayer (Wood et al., 2011), and theoretical considerations put forth by Giang and Schick (2014) support these findings. Cholesterol, it seems, prefers the high negative curvature offered by the inner bilayer leaflet, in effect lowering the bilayer's bending free energy. Its asymmetric distribution has also indirectly been observed in monounsaturated PC lipids (Kučerka et al. 2009). However, in those studies they were not able to resolve in which bilayer leaflet cholesterol was most abundant. 
Coarse-grain MD simulations have also produced some interesting results. For example, Yesylevskyy and Demchenko (2012) showed that cholesterol distributes asymmetrically in a lipid bilayer after $\sim 10 \mu \mathrm{s}$. Of note, is that its equilibrium distribution was dependent on the individual bilayer leaflet chemical composition, highlighting again the importance of lipid diversity in biomembranes.

\subsection{Bilayer Leaflet Re-distribution}

As mentioned, in erythrocytes and nucleated cells cholesterol is believed to be enriched on the cytosolic leaflet of the plasma membrane (negative curvature), which when one considers the typical membrane content of cholesterol (40 mol\%) implies a cholesterol-to-lipid ratio of greater than 1 (van Meer, 2011). This mol\% value is near the solubility limit of cholesterol in PC bilayers. Thus a continually balanced redistribution of cholesterol between leaflets is key to maintaining an adequate liquid $\mathrm{L}_{o}$ phase. Asymmetry is stabilized by the relatively quick - compared to lipids with their larger and/or more polar headgroups - flip-flop rate of cholesterol across the lipid bilayer. In many cases, however, protein intervention is needed to shuttle cholesterol across the bilayer (van Meer, 2011).

The flip-flop rates of cholesterol have been determined by a number of different experimental methods and by MD simulations, which have proven very valuable due to the complexity of probe-free studies. MD studies performed by Jo et al. (2010) and Bennett et al. (2009) yielded consistent average single molecule flip-flop ranging from $1.4 \mathrm{~ms}$ to 80 ns - depending on temperature and membrane composition. The prevailing trend in those studies is that cholesterol flip-flop increases with membrane disorder, which in turn increases with temperature and unsaturated lipid content, but decreases with lipid chain length. The thinner and more disordered lipid bilayers experience a larger degree of cholesterol interdigitation between the two bilayer leaflets - as their hydroxyl's hydrogen bonding partners are closer to the bilayer center - and are significantly more permeable to water. These ¿waters cause cholesterol to reorient into the nonpolar bilayer center (Figure 4). Kučerka et al. (2008) speculate that these features create a locally polar environment, promoting cholesterol flip-flop and stabilizing cholesterol's hydroxyl group within the nonpolar bilayer core. 

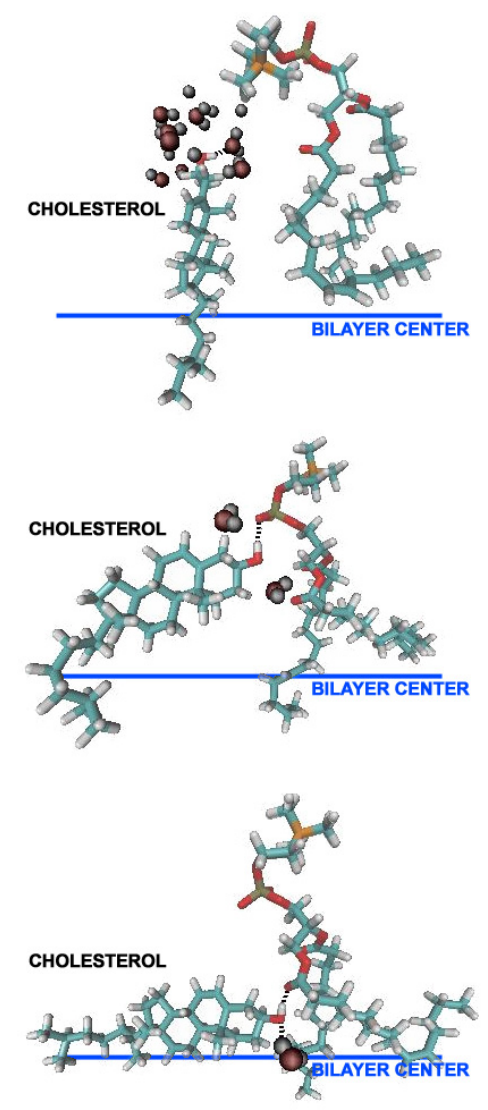

Figure 4: Sequential snapshots show dynamically changing interactions of cholesterol and lipids during flipflop. Cholesterol's reorientation is promoted by changing its hydrogen bonding from the lipid's phosphate to its carbonyl, a moiety that is significantly closer to the bilayer center (suggested by the blue line). Cholesterol's hydroxyl is accompanied by water molecules that further facilitate this transition. Figure adapted from Kučerka et al. (2008)

Experimentally determined cholesterol flip-flop rates are often higher than predicted by MD. A 1981 study using cholesterol oxidase to monitor cholesterol flip-flop put an upper limit of 1 minute on flip-flop half-time (Backer and Dawidowicz, 1981). This value is consistent with more recent data from Leventis and Silvius (2001) who determined a 1-2 minute flipflop half-time using ${ }^{3} \mathrm{H}$ labeled cholesterol partitioning studies. Other estimates of cholesterol flip-flop are on the order of seconds to nanoseconds (Steck et al., 2002; Bruckner et al., 2009; Hamilton, 2003; Endress et al., 2002). In contrast, a recent neutron scattering study of cholesterol flip-flop has yielded a half-time of $200 \mathrm{~min}$, highlighting the need for further study regarding this subject (Garg et al., 2011). 


\section{Cholesterol's Lateral Organization and Phospholipid Phase Behaviour}

It is well known that cholesterol has a significant effect on the phase behaviour of phosophlipid bilayers (Figure 5). Single component phospholipid bilayers exist, to a first approximation, in two different phases: the low temperature gel phase $\left(\mathrm{L}_{\beta}\right)$ and the high temperature liquid crystalline phase $\left(\mathrm{L}_{\alpha}\right)$. The $\mathrm{L}_{\beta}$ phase is characterized by high segmental fatty acid chain ordering (i.e., lipid hydrocarbon chains are almost fully extended) and the phospholipids are well-ordered in the two-dimensional plane of the bilayer (Smith et al., 1988; Katsaras et al., 1992; Raghunathan and Katsaras, 1995; Heberle and Feigenson, 2011). The transition from $\mathrm{L}_{\beta}$ to the higher temperature $\mathrm{L}_{\alpha}$ phase is a first order transition (Heberle and Feigenson, 2011), with the first direct observation of phase co-existence in a single component system made by Armstrong et al. (2012). Compared to the $\mathrm{L}_{\beta}$ phase, the $\mathrm{L}_{\alpha}$ phase exhibits increased hydrocarbon chain disorder (trans/gauche) - causing the chains to sample a larger conformation space - experiences the loss of two-dimensional long range order, and the lipids undergo increased lateral diffusion (Ipsen et al., 1987; Heberle and Feigenson, 2011; Kučerka et al. 2011; Rubenstein et al., 1979). However, when cholesterol is introduced to these single component lipid systems, an entirely new phase is introduced, namely the so-called liquid ordered phase $\left(\mathrm{L}_{o}\right)$ - the liquid crystalline $\mathrm{L}_{\alpha}$ phase is now termed the liquid disordered phase $\left(\mathrm{L}_{d}\right)$, although not entirely correct. The $\mathrm{L}_{o}$ is unique in that it has characteristics of both the $\mathrm{L}_{\beta}$ and $\mathrm{L}_{\alpha}$ phases. For example, in the $\mathrm{L}_{o}$ phase the lipids do not form an extended two-dimensional hydrocarbon chain lattice in the plane of the bilayer plane, yet the lipid acyl chains exhibit a high degree of chain segmental ordering and undergo relatively fast lipid lateral diffusion (Heberle and Feigenson, 2011; Veatch and Keller, 2005; Kahya et al., 2003, Ipsen et al., 1987). It is this so-called cholesterol rich $\mathrm{L}_{o}$ phase that is believed to provide the platform for functional domains or lipid rafts.

The existence of lipid rafts in living systems has been contentious, with a plethora of work devoted to their study over the last 30 years (Simons and Toomre, 2000; Hancock, 2006; Lingwood and Simons, 2010; Ipsen et al., 1987). Lipid raft compositions have been developed using ternary (de Almeida et al., 2003; Feigenson and Buboltz, 2001) and quaternary lipid mixtures, and cholesterol (Konyakhina et al., 2013). Studies of these lipid mixtures, and the resultant phase diagrams, have allowed for the identification of different phases (i.e., $\mathrm{L}_{o}$ and 


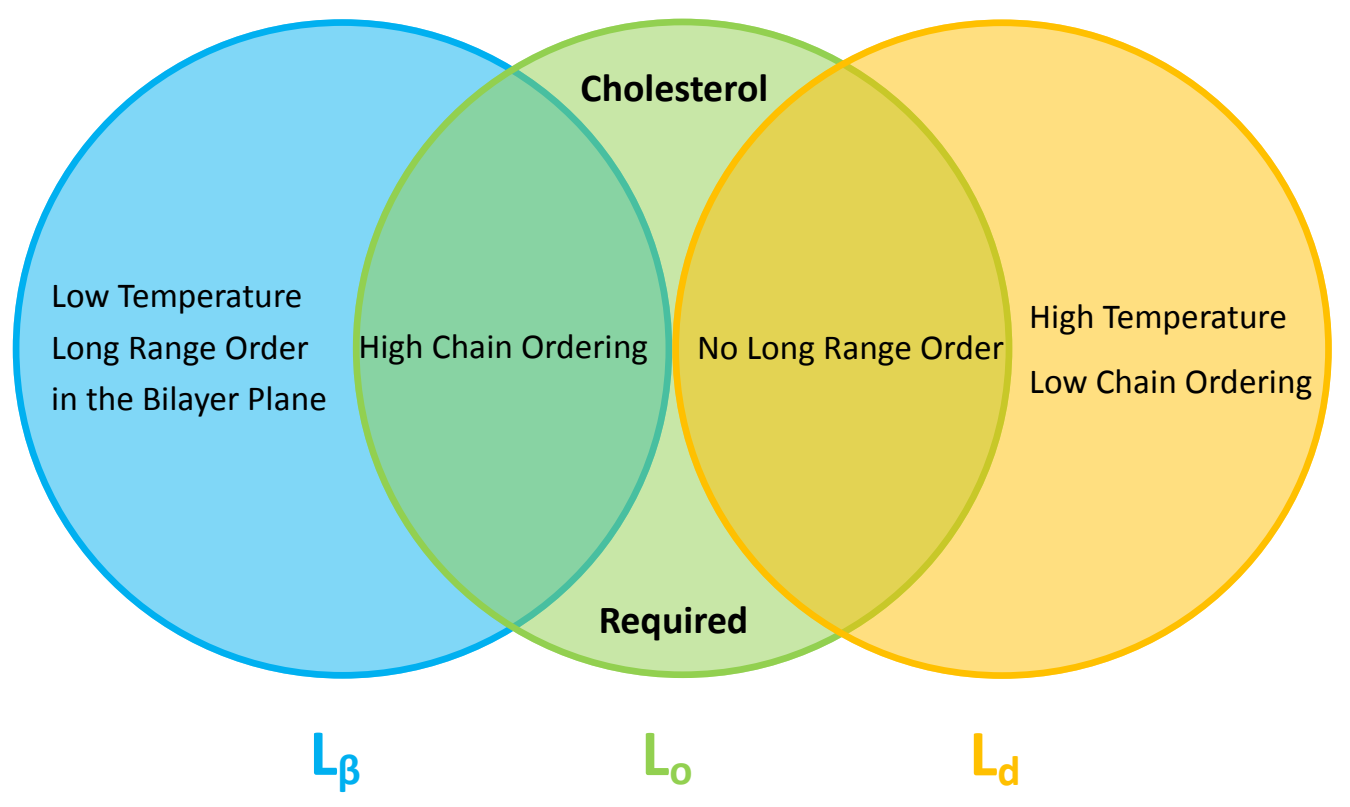

Figure 5: Venn diagram illustrating the properties shared between the liquid ordered $\left(\mathrm{L}_{o}\right)$ (contains cholesterol), gel $\left(\mathrm{L}_{\beta}\right)$ and liquid disordered $\left(\mathrm{L}_{d}\right)$ phases. Figure reproduced from Marquardt et al. (2015b)

$\left.\mathrm{L}_{d}\right)$ as a function of cholesterol content. To digress for a moment, Armstrong et al. (2013), however, recently observed the existence of cholesterol induced lipid domains in a nominally single phase lipid bilayer (i.e., DMPC). Importantly, the dynamical properties imparted by cholesterol to this $\mathrm{L}_{o}$ phase were rather unique, in that the observed nanoscale dynamics amplified certain properties of the $\mathrm{L}_{\beta}$ and $\mathrm{L}_{d}$ phases (Meinhardt et al., 2013; Shlomovitz et al. 2014; Toppozini et al., 2014). Specifically, the nanoscopic cholesterol containing domains appeared softer than $\mathrm{L}_{d}$ bilayers but more ordered than even $\mathrm{L}_{\beta}$ bilayers Armstrong et al. 2012). This amplification ability by cholesterol is thought to be an important driver in raft formation.

Heberle et al. (2013) studied the presence and size of nanoscopic domains in $60 \mathrm{~nm}$ diameter ULVs made up of a saturated phospholipid, a variable ratio of mono- and di-unsaturated phospholipids, and a constant mol\% of cholesterol. As the amount of the di-unsaturated lipid was increased (i.e., mono-unsaturated lipid decreased), domain size and bilayer thickness mismatch between the $\mathrm{L}_{d}$ and $\mathrm{L}_{o}$ both increased. This observation of increasing thickness mismatch with increasing domain diameter is consistent with theories espousing line tension as the driving force behind domain formation (Heberle et al., 2013; Kuzmin et al. 
2005; Frolov et al., 2006). These experiments, carried out at constant temperature, also lend insight as to how functional domains in biological membranes may be regulated, i.e., by altering the membrane's chemical composition.

\section{Concluding Remarks}

Cholesterol is an important precursor for the biosynthesis of certain hormones and vitamins, and is an essential biomolecule of animal cell membranes. In this review we have highlighted some of the effects that cholesterol has on model membranes. In turn, the "altered" membranes then influence the energetics of protein conformational transitions due to interactions between the protein's hydrophobic domains and the bilayers acyl chain region (Andersen and Koeppe, 2007). A better understanding of cholesterol's significance in membranes will eventually help us elucidate some of the open structure-function questions currently debated in biology.

\section{Acknowledgment}

DM thanks Georg Pabst for his support. NK is supported through the VEGA grant 1/1224/12 and collaborative SR-JINR program under theme 04-4-1121-2015/2017, JK is supported through the Scientific User Facilities Division of the DOE Office of Basic Energy Sciences under US DOE Contract No. DE-AC05-00OR22725.

\section{References}

Aittoniemi, J., Róg, T., Niemelä, P., Pasenkiewicz-Gierula, M., Karttunen, M., Vattulainen, I., 2006. Tilt: major factor in sterols' ordering capability in membranes. J. Phys. Chem. B. 110 (51), 25562-25564.

Andersen, O. S., Koeppe, R. E., 2007. Bilayer thickness and membrane protein function: an energetic perspective. Annu. Rev. Biophys. Biomol. Struct. 36, 107-130.

Armstrong, C. L., Barrett, M. A., Toppozini, L., Kučerka, N., Yamani, Z., Katsaras, J., Fragneto, G., Rheinstaedter, M. C., 2012. Co-existence of gel and fluid lipid domains in single-component phospholipid membranes. Soft Matter 8 (17), 4687-4694. 
Armstrong, C. L., Marquardt, D., Dies, H., Kučerka, N., Yamani, Z., Harroun, T. A., Katsaras, J., Shi, A. C., Rheinstaedter, M. C., 2013. The observation of highly ordered domains in membranes with cholesterol. PLoS One 8 (6).

Azzi, A., 2007. Molecular mechanism of alpha-tocopherol action. Free Radicals Biol. Med. $43(1), 16-21$.

Backer, J. M., Dawidowicz, E. A., 1981. Transmembrane movement of cholesterol in small unilamellar vesicles detected by cholesterol oxidase. J. Biol. Chem. 256 (2), 586-588.

Bartels, T., Lankalapalli, R. S., Bittman, R., Beyer, K., Brown, M. F., 2008. Raftlike mixtures of sphingomyelin and cholesterol investigated by solid-state $2 \mathrm{~h} \mathrm{nmr} \mathrm{spectroscopy.} \mathrm{J.}$ Am. Chem. Soc. 130 (44), 14521-14532.

Bennett, W. F. D., MacCallum, J. L., Hinner, M. J., Marrink, S. J., Tieleman, D. P., 2009. Molecular view of cholesterol flip-flop and chemical potential in different membrane environments. J. Am. Chem. Soc. 131 (35), 12714-12720.

Brown, A. J., Galea, A. M., 2010. Cholesterol as an evolutionary response to living with oxygen. Evolution (Hoboken, NJ, U. S.) 64 (7), 2179-2183.

Brown, M. S., Goldstein, J. L., 1992. A receptor-mediated pathway for cholesterol homeostasis. In: Lindsten, J. (Ed.), Nobel lectures: including presentation speeches and laureates' biographies. Nobel lecture. World Scientific, Singapore, pp. 284-324.

Bruckner, R. J., Mansy, S. S., Ricardo, A., Mahadevan, L., Szostak, J. W., 2009. Flip-flopinduced relaxation of bending energy: Implications for membrane remodeling. Biophys. J. 97 (12), 3113-3122.

Brzustowicz, Stillwell, W., Wassall, 1999. Molecular organization of cholesterol in polyunsaturated phospholipid membranes: a solid state h-2 nmr investigation. FEBS Lett. 451 (2), $197-202$.

Brzustowicz, M. R., Cherezov, V., Caffrey, M., Stillwell, W., Wassall, S. R., 2002a. Molecular organization of cholesterol in polyunsaturated membranes: Microdomain formation. Biophys. J. 82 (1), 285-298. 
Brzustowicz, M. R., Cherezov, V., Zerouga, M., Caffrey, M., Stillwell, W., Wassall, S. R., 2002b. Controlling membrane cholesterol content. a role for polyunsaturated (docosahexaenoate) phospholipids. Biochemistry 41 (41), 12509-12519.

Bunge, A., Müller, P., Stöckl, M., Herrmann, A., Huster, D., 2008. Characterization of the ternary mixture of sphingomyelin, popc, and cholesterol: support for an inhomogeneous lipid distribution at high temperatures. Biophys. J. 94 (7), 2680-2690.

Chiu, S. W., Jakobsson, E., Mashl, R. J., Scott, H. L., 2002. Cholesterol-induced modifications in lipid bilayers: A simulation study. Biophys. J. 83 (4), 1842-1853.

Davis, J. H., 1983. The description of membrane lipid conformation, order and dynamics by 2h-nmr. Biochim. Biophys. Acta, Rev. Biomembr. 737 (1), 117-171.

de Almeida, R. F., Fedorov, A., Prieto, M., 2003. Sphingomyelin/phosphatidylcholine/cholesterol phase diagram: Boundaries and composition of lipid rafts. Biophys. J. 85 (4), 2406-2416.

Dufourc, E. J., Parish, E. J., Chitrakorn, S., Smith, I., 1984. Structural and dynamical details of cholesterol lipid interaction as revealed by deuterium nmr. Biochemistry 23 (25), 60626071.

Endress, E., Heller, H., Casalta, H., Brown, M. F., Bayerl, T. M., 2002. Anisotropic motion and molecular dynamics of cholesterol, lanosterol, and ergosterol in lecithin bilayers studied by quasi-elastic neutron scattering $\uparrow$. Biochemistry 41 (43), 13078-13086.

Feigenson, G. W., Buboltz, J. T., 2001. Ternary phase diagram of dipalmitoyl-pc/dilauroylpc/cholesterol: nanoscopic domain formation driven by cholesterol. Biophys. J. 80 (6), 2775-2788-.

Franks, N. P., Lieb, W. R., 1979. The structure of lipid bilayers and the effects of general anaesthetics. J. Mol. Biol. 133 (4), 469-500.

Frolov, V., Chizmadzhev, Y. A., Cohen, F. S., Zimmerberg, J., 2006. "entropic traps" in the kinetics of phase separation in multicomponent membranes stabilize nanodomains. Biophys. J. 91 (1), 189-205. 
Gallová, J., Klacsová, M., Devínsky, F., Balgavý, P., 2015. Partial volumes of cholesterol and monounsaturated diacylphosphatidylcholines in mixed bilayers. Chem. Phys. Lipids $190,1-8$.

Garg, S., Porcar, L., Woodka, A. C., Butler, P. D., Perez-Salas, U., 2011. Noninvasive neutron scattering measurements reveal slower cholesterol transport in model lipid membranes. Biophys. J. 101 (2), 370-377.

Gawrisch, K., Soubias, O., 2008. Structure and dynamics of polyunsaturated hydrocarbon chains in lipid bilayers-significance for gpcr function. Chem. Phys. Lipids 153 (1), 64-75.

Giang, H., Schick, M., 2014. How cholesterol could be drawn to the cytoplasmic leaf of the plasma membrane by phosphatidylethanolamine. Biophys. J. 107 (10), 2337-2344.

Gilbert, D. B., Tanford, C., Reynolds, J. A., 1975. Cholesterol in aqueous-solution - hydrophobicity and self association. Biochemistry 14 (2), 444-448.

Girotti, A. W., 2001. Photosensitized oxidation of membrane lipids: Reaction pathways, cytotoxic effects, and cytoprotective mechanisms. J. Photochem. Photobiol., B 63 (1-3), $103-113$.

Greenwood, A. I., Tristram-Nagle, S., Nagle, J. F., 2006. Partial molecular volumes of lipids and cholesterol. Chem. Phys. Lipids 143 (1-2), 1-10.

Haberlan, M. E., Reynolds, J. A., 1973. Self-association of cholesterol in aqueous-solution. Proc. Natl. Acad. Sci. U. S. A. 70 (8), 2313-2316.

Hamilton, J. A., 2003. Fast flip-flop of cholesterol and fatty acids in membranes: implications for membrane transport proteins. Curr. Opin. Lipidol. 14 (3), 263-271.

Hancock, J. F., 2006. Lipid rafts: contentious only from simplistic standpoints. Nat. Rev. Mol. Cell Biol. 7 (6), 456-462.

Harroun, T., Wignall, G., Katsaras, J., 2006a. Neutron scattering for biology: 1-18. In: Jörg Fitter, Thomas Gutberlet, John Katsaras (Eds.), Neutron Scattering in Biology Techniques and Applications. Springer, pp. 1-18. 
Harroun, T. A., Katsaras, J., Wassall, S. R., 2006b. Cholesterol hydrozyl group is found to reside in the center of a polyunsaturated lipid membrane. Biochemistry 45, 1227-1233.

Harroun, T. A., Katsaras, J., Wassall, S. R., 2008. Cholesterol is found to reside in the center of a polyunsaturated lipid membrane. Biochemistry 47 (27), 7090-7096.

Heberle, F. A., Feigenson, G. W., 2011. Phase separation in lipid membranes. Cold Spring Harbor Perspect. Biol. 3 (4).

Heberle, F. A., Petruzielo, R. S., Pan, J., Drazba, P., Kučerka, N., Standaert, R. F., Feigenson, G. W., Katsaras, J., 2013. Bilayer thickness mismatch controls domain size in model membranes. J. Am. Chem. Soc. 135 (18), 6853-6859.

Hofsäß, C., Lindahl, E., Edholm, O., 2003. Molecular dynamics simulations of phospholipid bilayers with cholesterol. Biophys. J. 84 (4), 2192-2206.

Ipsen, J. H., Karlstrom, G., Mouritsen, O. G., Wennerstrom, H., Zuckermann, M. J., 1987. Phase equilibria in phosphatidylcholine-cholesterol system. Biochim. Biophys. Acta 905, $162-172$.

Jackman, C. S., Davis, P. J., Morrow, M. R., Keough, K. M. W., 1999. Effect of cholesterol on the chain-ordering transition of 1-palmitoyl-2-arachidonoyl phosphatidylcholine 103 (42), 8830-8836.

Jo, S., Rui, H., Lim, J. B., Klauda, J. B., Im, W., 2010. Cholesterol flip-flop: insights from free energy simulation studies. J. Phys. Chem. B. 114 (42), 13342-13348.

Kahya, N., Scherfeld, D., Bacia, K., Poolman, B., Schwille, P., 2003. Probing lipid mobility of raft-exhibiting model membranes by fluorescence correlation spectroscopy. J.Biol. Chem. 278 (30), 28109-28115.

Katsaras, J., Yang, D. S.-C., Epand, R. M., 1992. Fatty acid chain tilt angles and directions in dipalmitoyl phosphatidylcholine bilayers. Biophys. J. 63, 1170.

Kessel, A., Ben-Tal, N., May, S., 2001. Interactions of cholesterol with lipid bilayers: The preferred configuration and fluctuations. Biophys. J. 81 (2), 643-658. 
Khelashvili, G., Harries, D., 2013. How cholesterol tilt modulates the mechanical properties of saturated and unsaturated lipid membranes. J. Phys. Chem. B.

Konyakhina, T. M., Wu, J., Mastroianni, J. D., Heberle, F. A., Feigenson, G. W., 2013. Phase diagram of a 4-component lipid mixture: Dspc/dopc/popc/chol. Biochim. Biophys. Acta 1828 (9), 2204-2214.

Kristiana, I., Luu, W., Stevenson, J., Cartland, S., Jessup, W., Belani, J. D., Rychnovsky, S. D., Brown, A. J., 2012. Cholesterol through the looking glass: ability of its enantiomer also to elicit homeostatic responses. J. Biol. Chem. 287 (40), 33897-33904.

Kučerka, N., Marquardt, D., Harroun, T. A., Nieh, M.-P., Wassall, S. R., Jong, D. H. d., Schäfer, L. V., Marrink, S. J., Katsaras, J., 2010. Cholesterol in bilayers with pufa chains: doping with dmpc or popc results in sterol reorientation and membrane-domain formation. Biochemistry 49 (35), 7485-7493.

Kučerka, N., Nieh, M.-P., Katsaras, J., 2011. Fluid phase lipid areas and bilayer thicknesses of commonly used phosphatidylcholines as a function of temperature. Biochim. Biophys. Acta 1808, 2761-2771.

Kučerka, N., Nieh, M.-P., Pencer, J., Sachs, J. N., Katsaras, J., 2009. What determines the thickness of a biological membrane. Gen. Physiol. Biophys. 28 (2), 117-125.

Kučerka, N., Pencer, J., Nieh, M.-P., Katsaras, J., 2007. Influence of cholesterol on the bilayer properties of monounsaturated phosphatidylcholine unilamellar vesicles. Eur. Phys. J. E: Soft Matter Biol. Phys. 23 (3), 247-254.

Kučerka, N., Perlmutter, J. D., Pan, J., Tristram-Nagle, S., Katsaras, J., Sachs, J. N., 2008. The effect of cholesterol on short- and long-chain monounsaturated lipid bilayers as determined by molecular dynamics simulations and x-ray scattering. Biophys. J. 95 (6), $2792-2805$.

Kuzmin, P. I., Akimov, S. A., Chizmadzhev, Y. A., Zimmerberg, J., Cohen, F. S., 2005. Line tension and interaction energies of membrane rafts calculated from lipid splay and tilt. Biophys. J. 88 (2), 1120-1133. 
Lafleur, M., Bloom, M., Cullis, P. R., 1990. Modulation of the orientational order profile of the lipid acyl chain in the 1 alpha phase. Eur. Biophys. J. 19 (2), 55-62.

Lange, Y., Ramos, B. V., 1983. Analysis of the distribution of cholesterol in the intact cell. J. Biol. Chem. 258 (24), 5130-5134.

Leng, X., Kinnun, J. J., Marquardt, D., Ghefli, M., Kučerka, N., Katsaras, J., Atkinson, J., Harroun, T. A., Feller, S. E., Wassall, S. R., 2015. $\alpha$-tocopherol is well designed to protect polyunsaturated phospholipids: Md simulations 109 (8), 1608-1618.

Leonard, A., Escrive, C., Laguerre, M., Pebay-Peyroula, E., Neri, W., Pott, T., Katsaras, J., Dufourc, E. J., 2001. Location of cholesterol in dmpc membranes. a comparative study by neutron diffraction and molecular mechanics simulation. Langmuir 17 (6), 2019-2030.

Leventis, R., Silvius, J. R., 2001. Use of cyclodextrins to monitor transbilayer movement and differential lipid affinities of cholesterol. Biophys. J. 81 (4), 2257-2267.

Lingwood, D., Simons, K., 2010. Lipid rafts as a membrane-organizing principle. Science 327 (5961), 46-50.

Marquardt, D., Geier, B., Pabst, G., 2015a. Asymmetric lipid membranes: towards more realistic model systems. Membranes 5 (2), 180-196.

Marquardt, D., Harroun, T., 2014. Locations of small biomolecules in model membranes. In: Pabst, G., Kučerka, N., Nieh, M.-P., Katsaras, J. (Eds.), Liposomes, Lipid Bilayers and Model Membranes. CRC Press, pp. 199-216.

Marquardt, D., Heberle, F. A., Nickels, J. D., Pabst, G., Katsaras, J., 2015b. On scattered waves and lipid domains: Detecting membrane rafts with x-rays and neutrons. Soft Matter 11 (47), 9055-9072.

Marquardt, D., Kučerka, N., Katsaras, J., Harroun, T. A., 2015c. $\alpha$-tocopherol's location in membranes is not affected by their composition. Langmuir 31 (15), 4464-4472. 
Marquardt, D., van Oosten, B., Heberle, F. A., Kučerka, N., Wassall, S., Standaert, R., Katsaras, J., Harroun, T. A., 2015d. Hydrocarbon thickness dictates cholesterol's location, orientation and motion in a phospholipid bilayer 108 (2), 86a.

Marquardt, D., Williams, J. A., Kinnun, J. J., Kučerka, N., Atkinson, J., Wassall, S. R., Katsaras, J., Harroun, T. A., 2014. Dimyristoyl phosphatidylcholine: a remarkable exception to $\alpha$-tocopherol's membrane presence. J. Am. Chem. Soc. 136 (1), 203-210.

Marquardt, D., Williams, J. A., Kučerka, N., Atkinson, J., Wassall, S. R., Katsaras, J., Harroun, T. A., 2013. Tocopherol activity correlates with its location in a membrane: A new perspective on the antioxidant vitamin e. J. Am. Chem. Soc. 135 (20), 7523-7533.

Marsan, M. P., Muller, I., Ramos, C., Rodriguez, F., Dufourc, E. J., Czaplicki, J., Milon, A., 1999. Cholesterol orientation and dynamics in dimyristoylphosphatidylcholine bilayers: A solid state deuterium nmr analysis. Biophys. J. 76 (1, Part 1), 351-359.

Meinhardt, S., Vink, R. L. C., Schmid, F., 2013. Monolayer curvature stabilizes nanoscale raft domains in mixed lipid bilayers. Proc. Natl. Acad. Sci. U.S.A. 110 (12), 4476-4481.

Mihailescu, M., Soubias, O., Worcester, D., White, S. H., Gawrisch, K., 2011. Structure and dynamics of cholesterol-containing polyunsaturated lipid membranes studied by neutron diffraction and nmr. J. Membr. Biol. 239 (1-2), 63-71.

Miyoshi, T., Lönnfors, M., Peter Slotte, J., Kato, S., 2014. A detailed analysis of partial molecular volumes in dppc/cholesterol binary bilayers. Biochim. Biophys. Acta 1838 (12), 3069-3077.

Mouritsen, O. G., 2005. Life - as a matter of fat: The emerging science of lipidomics. Frontiers collection. Springer, Berlin.

Murari, R., Murari, M. P., Baumann, W. J., 1986. Sterol orientations in phosphatidylcholine liposomes as determined by deuterium nmr. Biochemistry 25 (5), 1062-1067.

Myant, N. B., 1981. The Biology of Cholesterol and Related Steroids. Elsevier Science, Burlington. 
Nickels, J. D., Smith, J. C., Cheng, X., 2015. Lateral organization, bilayer asymmetry, and inter-leaflet coupling of biological membranes. Chem. Phys. Lipids.

Niu, S. L., Litman, B. J., 2002. Determination of membrane cholesterol partition coefficient using a lipid vesicle-cyclodextrin binary system: Effect of phospholipid acyl chain unsaturation and headgroup composition. Biophys. J. 83 (6), 3408-3415.

Oldfield, E., Meadows, M., Rice, D., Jacobs, R., 1978. Spectroscopic studies of specifically deuterium labeled membrane systems. nuclear magnetic resonance investigation of the effects of cholesterol in model systems. Biochemistry 17 (14), 2727-2740.

Pan, J., Cheng, X., Heberle, F. A., Mostofian, B., Kučerka, N., Drazba, P., Katsaras, J., 2012. Interactions between ether phospholipids and cholesterol as determined by scattering and molecular dynamics simulations. J. Phys. Chem. B. 116 (51), 14829-14838.

Pan, J., Mills, T. T., Tristram-Nagle, S., Nagle, J. F., 2008. Cholesterol perturbs lipid bilayers nonuniversally. Phys. Rev. Lett. 100 (19).

Pandey, B. N., Mishra, K. P., 1999. Radiation induced oxidative damage modification by cholesterol in liposomal membrane. Radiat. Phys. Chem. 54 (5), 481-489.

Papanikolaou, A., Papafotika, A., Murphy, C., Papamarcaki, T., Tsolas, O., Drab, M., Kurzchalia, T. V., Kasper, M., Christoforidis, S., 2005. Cholesterol-dependent lipid assemblies regulate the activity of the ecto-nucleotidase cd39. J. Biol. Chem. 280 (28), $26406-26414$.

Parasassi, T., Giusti, A. M., Raimondi, M., Ravagnan, G., Sapora, O., Gratton, E., 1995. Cholesterol protects the phospholipid bilayer from oxidative damage. Free Radicals Biol. Med. 19 (4), 511-516.

Petrie, R. J., Schnetkamp, P. P. M., Patel, K. D., Awasthi-Kalia, M., Deans, J. P., 2000. Transient translocation of the b cell receptor and src homology 2 domain-containing inositol phosphatase to lipid rafts: Evidence toward a role in calcium regulation. J. Immunol. 165 (3), 1220-1227. 
Raghunathan, V., Katsaras, J., 1995. Structure of the l(c') phase in a hydrated lipid multilamellar system. Phys. Rev. Lett. 74 (22), 4456-4459.

Róg, T., Pasenkiewicz-Gierula, M., 2001. Cholesterol effects on the phospholipid condensation and packing in the bilayer: A molecular simulation study. FEBS Lett. 502 (1-2), $68-71$.

Róg, T., Pasenkiewicz-Gierula, M., 2003. Effects of epicholesterol on the phosphatidylcholine bilayer: A molecular simulation study. Biophys. J. 84 (3), 1818-1826.

Rubenstein, J. L., Smith, B. A., McConnell, H. M., 1979. Lateral diffusion in binary mixtures of cholesterol and phosphatidylcholines. Proc. Natl. Acad. Sci. U.S.A. 76 (1), 15-18.

Seelig, J., 1977. Deuterium magnetic resonance: theory and application to lipid membranes. Q. Rev. Biophys. 10 (3), 353-418.

Seelig, J., Waespe-Sarcevic, N., 1978. Molecular order in cis and trans unsaturated phospholipid bilayers. Biochemistry 17 (16), 3310-3315.

Shaikh, Cherezov, V., Caffrey, M., Soni, S. P., LoCascio, D., Stillwell, W., Wassall, 2006. Molecular organization of cholesterol in unsaturated phosphatidylethanolamines: X-ray diffraction and solid state h-2 nmr reveal differences with phosphatidylcholines. J. Am. Chem. Soc. 128 (16), 5375-5383.

Shaikh, S. R., Kinnun, J. J., Leng, X., Williams, J. A., Wassall, S. R., 2015. How polyunsaturated fatty acids modify molecular organization in membranes: insight from nmr studies of model systems. Biochim. Biophys. Acta 1848 (1 Pt B), 211-219.

Shlomovitz, R., Maibaum, L., Schick, M., 2014. Macroscopic phase separation, modulated phases, and microemulsions: A unified picture of rafts. Biophysical Journal 106 (9), 19791985.

Shrivastava, S., Haldar, S., Gimpl, G., Chattopadhyay, A., 2009. Orientation and dynamics of a novel fluorescent cholesterol analogue in membranes of varying phase 113 (13), 44754481. 
Simons, K., Toomre, D., 2000. Nat. Rev. Mol. Cell Biol. 1 (1), 31-39.

Smith, G. S., Sirota, E. B., Safinya, C. R., Clark, N. A., 1988. Structure of the $1_{\beta}$ phase in a hydrated phosphatidylcholine multimembrane. Phys. Rev. Lett. 60, 813-816.

Smith, L. L., 1991. Another cholesterol hypothesis: Cholesterol as antioxidant. Free Radicals Biol. Med. 11 (1), 47-61.

Steck, T. L., Ye, J., Lange, Y., 2002. Probing red cell membrane cholesterol movement with cyclodextrin. Biophys. J. 83 (4), 2118-2125.

Stillwell, W., 2013. Chronology of membrane studies. In: Stillwell, W. (Ed.), An Introduction to Biological Membranes. Elsevier, San Diego, pp. 357-360.

Subczynski, W. K., Widomska, J., Feix, J. B., 2009. Physical properties of lipid bilayers from epr spin labeling and their influence on chemical reactions in a membrane environment. Free Radicals Biol. Med. 46 (6), 707-718.

Tieleman, D. P., Marrink, S.-J., 2006. Lipids out of equilibrium: energetics of desorption and pore mediated flip-flop. J. Am. Chem. Soc. 128 (38), 12462-12467.

Toppozini, L., Meinhardt, S., Armstrong, C. L., Yamani, Z., Kučerka, N., Schmid, F., Rheinstädter, M. C., 2014. Structure of cholesterol in lipid rafts. Physical Review Letters $113(22)$.

Traber, M. G., Atkinson, J., 2007. Vitamin e, antioxidant and nothing more. Free Radical Biol. Med. 43 (1), 4-15.

van Meer, G., 2011. Dynamic transbilayer lipid asymmetry. Cold Spring Harbor Perspect. Biol. 327, 3:a004671.

Veatch, S. L., Keller, S. L., 2005. Seeing spots: Complex phase behavior in simple membranes. Biochim. Biophys. Acta, Mol. Cell Res. 1746 (3), 172-185.

Vist, M. R., Davis, J. H., 1990. Phase equilibria of cholesterol/dipalmitoylphosphatidylcholine mixtures: Deuterium nuclear magnetic resonance and differential scanning calorimetry. Biochemistry 29 (2), 451-464. 
Wassall, S. R., Stillwell, W., 2008. Docosahexaenoic acid domains: the ultimate non-raft membrane domain. Chem. Phys. Lipids 153 (1), 57-63.

Westover, E. J., Covey, D. F., Brockman, H. L., Brown, R. E., Pike, L. J., 2003. Cholesterol depletion results in site-specific increases in epidermal growth factor receptor phosphorylation due to membrane level effects. studies with cholesterol enantiomers. J. Biol. Chem. 278 (51), 51125-51133.

Williams, J., Wassall, C. D., Kemple, M. D., Wassall, S. R., 2013. Dependence of cholesterolphospholipid affinity on degree of acyl chain unsaturation as determined by epr. Biophys. J. $104(2), 588$ a.

Wood, W. G., Igbavboa, U., Müller, W. E., Eckert, G. P., 2011. Cholesterol asymmetry in synaptic plasma membranes. J. Neurochem. 116 (5), 684-689.

Worcester, D. L., Franks, N. P., 1976. Structural analysis of hydrated egg lecithin and cholesterol bilayers ii. neutron diffraction. J. Mol. Biol. 100 (3), 359-378.

Yasuda, T., Kinoshita, M., Murata, M., Matsumori, N., 2014. Detailed comparison of deuterium quadrupole profiles between sphingomyelin and phosphatidylcholine bilayers. Biophys. J. 106 (3), 631-638.

Yeagle, P. L., 1993. The biophysics and cell biology of cholesterol: an hypothesis for the essential role of cholesterol in mammalian cells. In: Finegold, L. (Ed.), Cholesterol in membrane models. CRC Press, Boca Raton, Fla., pp. 1-12.

Yesylevskyy, S. O., Demchenko, A. P., 2012. How cholesterol is distributed between monolayers in asymmetric lipid membranes. Eur. Biophys. J. 41 (12), 1043-1054.

Zhang, Z., Lu, L., Berkowitz, M. L., 2008. Energetics of cholesterol transfer between lipid bilayers. J. Phys. Chem. B. 112 (12), 3807-3811. 

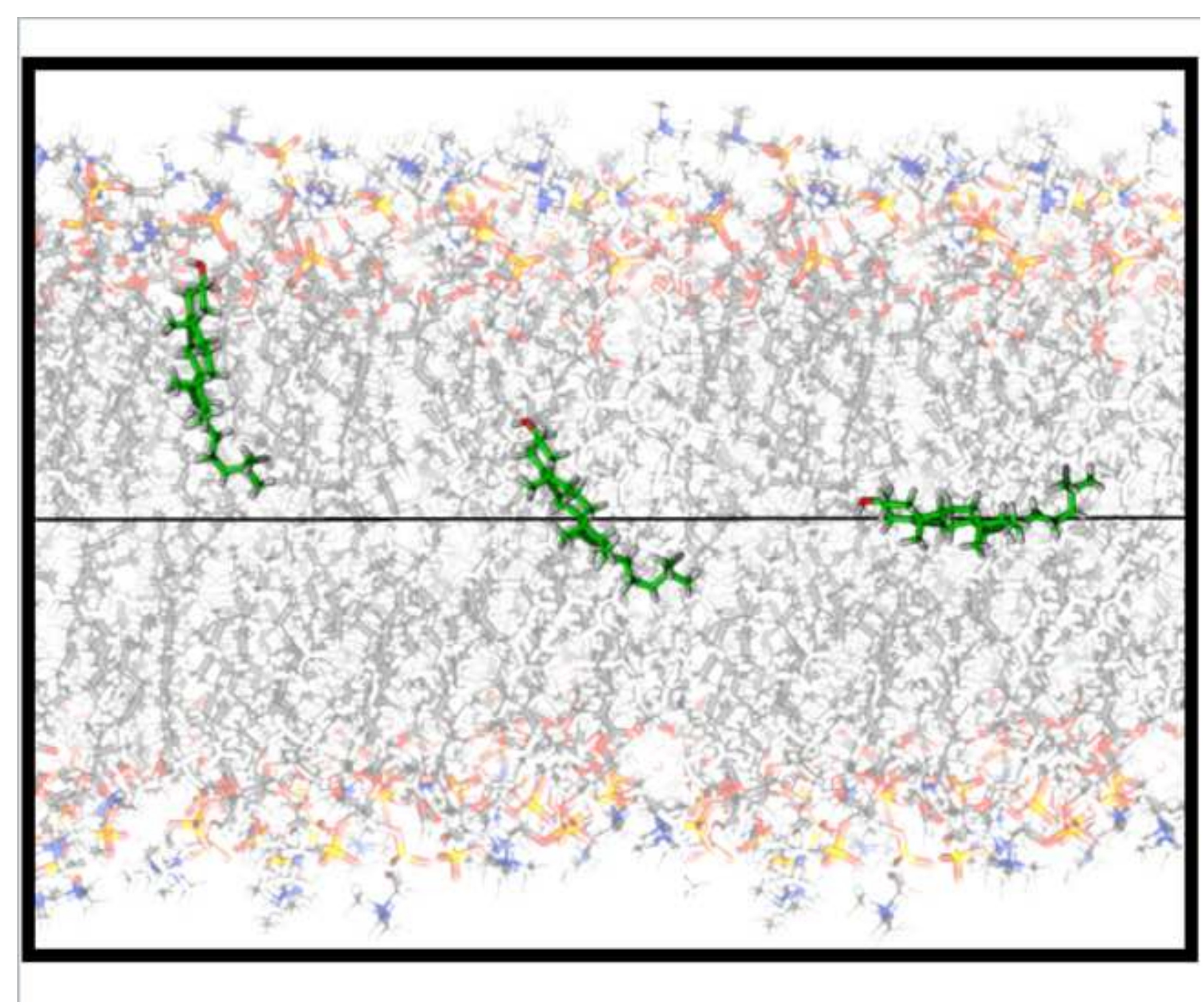